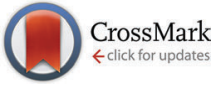

Cite this: Chem. Commun., 2015, 51, 11052

Received 1st April 2015,

Accepted 5th June 2015

DOI: $10.1039 / \mathrm{c} 5 \mathrm{cc} 02716 \mathrm{k}$

www.rsc.org/chemcomm

\section{Orthogonal breaking and forming of dynamic covalent imine and disulfide bonds in aqueous solution $\dagger$}

\author{
Michael E. Bracchi and David A. Fulton*
}

\begin{abstract}
Orthogonal bond-breaking and forming of dynamic covalent disulfide and imine bonds in aqueous solution is demonstrated. Through judicious choice of reaction partners and conditions, it is possible to cleave and reform selectively these bonds in the presence of each other in the absence of unwanted competing processes.
\end{abstract}

The design and study of functional systems of molecules is an area of interest within the growing field of systems chemistry. ${ }^{1}$ An approach to increase system complexity is to exploit multiple orthogonal supramolecular interactions, yet only in recent years have chemists made significant efforts ${ }^{2}$ to emulate this feature which is ubiquitous in natural systems. Orthogonal interactions in functional systems ${ }^{3}$ have been exploited in increasingly complex systems e.g. molecular machines, ${ }^{4}$ interlocked molecules ${ }^{5}$ and responsive materials, ${ }^{6}$ and to continue this development there is a clear need for well-understood orthogonal interactions.

Chemical bonds which can reversibly break and reform in response to stimuli are well-known ${ }^{7}$ in chemistry, and these so-called "dynamic covalent bonds" (DCBs) can be utilized as "modules", to introduce stimuli-responsiveness into functional systems. Of particular interest to us is reversible imine and disulfide bonds. Imine bonds are formed from the condensation of amines and carbonyls (Fig. 1a), and the position of the equilibrium is $\mathrm{pH}$ dependent, with work by Lehn demonstrating ${ }^{9}$ that the position of the imine equilibrium can be shifted from almost complete imine to starting materials over about three $\mathrm{pH}$ units. Redox-sensitive disulfide bonds can be reduced to their corresponding thiols in the presence of a reducing stimulus, and re-oxidized to form the disulfide (Fig. 1b). ${ }^{10}$ Since $\mathrm{pH}$ and redox can be controlled independent of each other it should be possible to selectively cleave and reform one of these bonds in the presence of the other, and thus these bonds can be considered to be orthogonal.\$ Assuming complete orthogonality, and considering

A Chemical Nanoscience Laboratory, School of Chemistry, Newcastle University, Bedson Building, Newcastle Upon Tyne, NE1 7RU, UK.

E-mail: david.fulton@ncl.ac.uk; Fax: +44 (0)191 208 6929;

Tel: +44 (0)191208 7065

$\dagger$ Electronic supplementary information (ESI) available. See DOI: 10.1039/c5cc02716k (a)
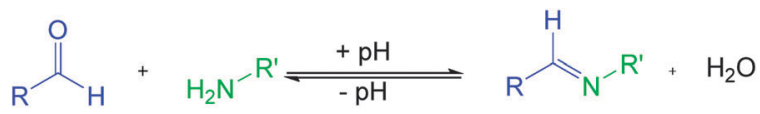

(b)
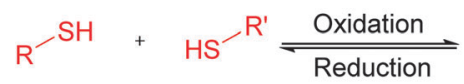

$R^{-S} S^{-S^{-}} R^{\prime}$

(c)

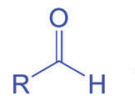

$H S^{-R^{\prime}}$

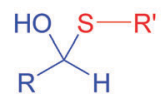

Fig. 1 (a) pH-sensitive imine formation and hydrolysis, (b) redox-sensitive disulfide formation and cleavage, (c) hemithioacetal formation.

only situations in which DCBs are in either "broken" or "formed" states, there are four distinct scenarios. It is convenient to map these states onto a four-node network (Fig. 2) where each node represents one of four possible scenarios regarding whether the disulfide and imine bonds are "broken" or "formed" and the vertices display the orthogonal stimuli required to drive the bond forming and breaking processes. The condition for orthogonality is that it is possible to successfully navigate between all nodes through the application of orthogonal stimuli with no undesired reactions occurring between the molecules in the mixture i.e. alternative combinations of the reaction partners are not detectable by ${ }^{1} \mathrm{H}$ NMR spectroscopy. In particular, the reaction between thiols and aldehydes to form hemithioacetals (Fig. 1c) was identified as a potential competing process, and our investigation showed that careful choice of reaction partners is necessary to avoid this problem.

In this work we investigate the orthogonality of the bond breaking and forming of imine and disulfide DCBs by establishing a set of conditions under which sequential application of stimuli allows interconversion between nodes within a 4-node network in the absence of unwanted, interfering processes. ${ }^{11}$ We also highlight how careful choice of reaction partners is required in order to avoid formation of undesired products and 'cross-talk' between our chosen DCB motifs.

As a redox-sensitive DCB, we chose the disulfide 1 whose quaternary ammonium groups impart water solubility. It is possible 


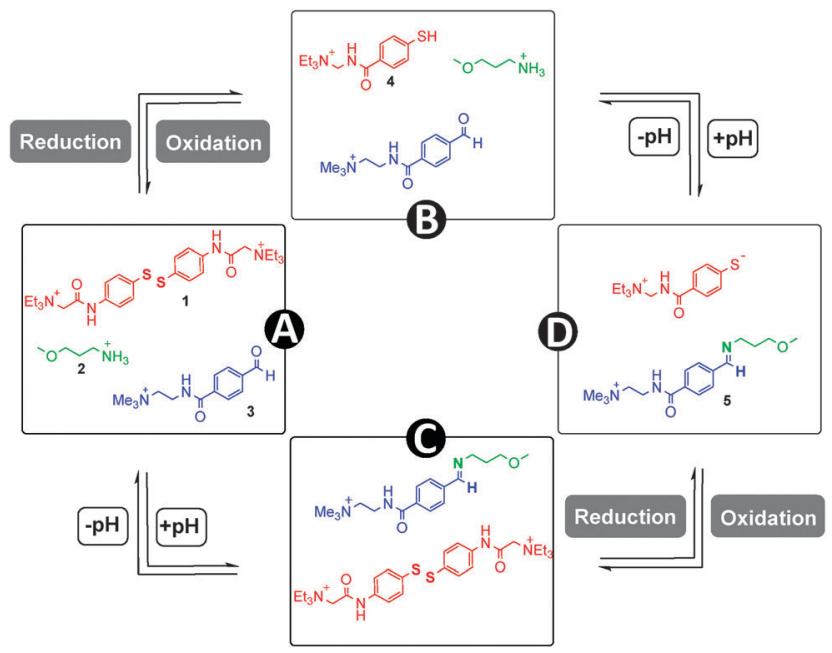

Fig. 2 Considering only situations in which imine and disulfide DCBs are in either "broken" or "formed" states, there are four distinct scenarios which can be mapped onto a four-node network. In node A, the disulfide bond is "formed" and the imine "broken". In node B both the disulfide and imine bonds are "broken". In node $C$ both the disulfide and imine bonds are "formed", and in node D the disulfide bond is "broken" the imine "formed". It is possible to successfully navigate between all nodes through the application of the appropriate orthogonal stimuli.

to reversibly interconvert this species with thiol 4 upon application of reducing and oxidising agents. As a pH-sensitive DCB we chose imine $\mathbf{5}$, which can be reversibly interconverted into its water-soluble reaction partners amine 2 and aldehyde 3 by modulation of $\mathrm{pH}$. The imine is formed almost exclusively at $\mathrm{pH}$ 12.0, and the reaction partners at $\mathrm{pH}$ 6.5.

The four-node network can be analyzed starting at any node, and for the sake of experimental simplicity we started at node A. A solution of disulfide 1, amine 2 and aldehyde 3 in $\mathrm{D}_{2} \mathrm{O}$ (15 mM of each of these three species) at $\mathrm{pH} 6.5$ was prepared and analyzed by ${ }^{1} \mathrm{H}$ NMR spectroscopy (Fig. 3a). The presence of aldehyde 3 is confirmed by a singlet at $\delta=9.9 \mathrm{ppm}$ and a pair of aromatic doublets at $\delta=7.7-8.0 \mathrm{ppm}$. Signals at $\delta=7.4 \mathrm{ppm}$ indicates the presence of the aromatic disulfide 1 . Importantly, the spectrum indicates disulfide $\mathbf{1}$ is stable at $\mathrm{pH}$ 6.5 and the absence of a signal at $\delta=8.4 \mathrm{ppm}$ indicates that there is no unwanted imine formed at this pH. Closer examination of this spectrum does reveal a second pair of doublets of extremely low intensity between $\delta=7.5-7.7 \mathrm{ppm}$ and a singlet at $\sim \delta=6.0 \mathrm{ppm}$ suggesting the presence of a small amount of hydrate. The hydrate of 3 exists merely as a "spectating", species in low concentration at $\mathrm{pH} 6.5$ and does not influence the orthogonality of the imine and disulfide bonds.

To drive the transition from node $\mathrm{A}$ to node $\mathrm{B}$, a reductive stimulus was applied through the addition of a slight excess of the organic reductant dithiothreitol (DTT). Analysis by ${ }^{1} \mathrm{H}$ NMR spectroscopy (Fig. 3b) reveals the appearance of a broad multiplet at $\delta=7.8 \mathrm{ppm}$ associated with thiol 4 and the disappearance of the pair of doublets at $\delta=7.3-7.5 \mathrm{ppm}$ associated with disulfide 1 . This observation indicates the successful and complete reduction of the disulfide $\mathbf{1}$ into thiol $\mathbf{4}$. The signals corresponding to
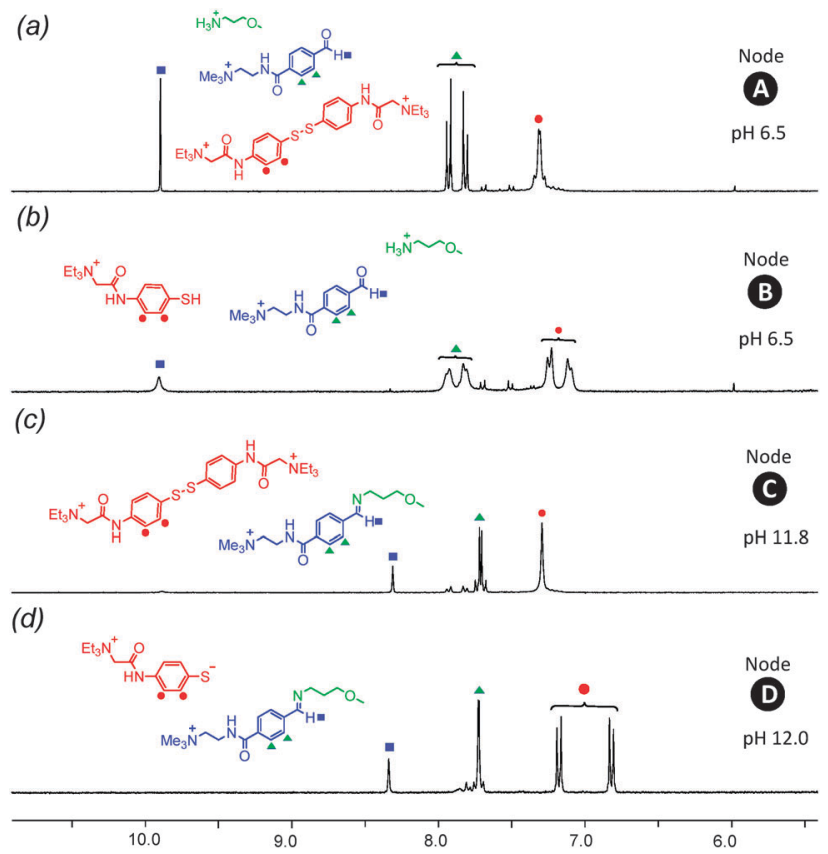

Fig. $3{ }^{1} \mathrm{H}$ NMR spectra $\left(300 \mathrm{MHz}, \mathrm{D}_{2} \mathrm{O}\right.$ ) collected at each 'node' in the 4-node network. (a) In node A, the disulfide bond is "formed" and the imine "broken". (b) In node B both the disulfide and imine bonds are "broken". (c) In node C both the disulfide and imine bonds are "formed", (d) in node D the disulfide bond is "broken" the imine "formed".

aldehyde 3 remain unchanged suggesting no unwanted hemithioacetal formation has occurred as through reaction of thiol 4 with aldehyde 3 . Furthermore, signals corresponding to amine 2 remain unchanged between nodes A to $\mathrm{B}$ (see $\mathrm{ESI} \dagger$ for expanded spectra), indicating no unwanted processes occur involving 2. A degree of unexpected spectra broadening was observed which further NMR work indicates is attributable to dynamic processes (see ESI $\dagger$ Fig. S7, S8 for full details), however, this spectrum is still sufficiently informative to fully support the conclusions drawn.

The transition from node $\mathrm{B}$ to node $\mathrm{D}$ was driven by applying an increase in $\mathrm{pH}$, serving as a stimulus to favor the condensation of 2 with 3 to form imine 5 . The $\mathrm{pH}$ was raised from 6.5 to 12.0 using $10 \mu \mathrm{l}$ aliquots of $0.1 \mathrm{M} \mathrm{NaOH}$, and subsequent analysis by ${ }^{1} \mathrm{H}$ NMR spectroscopy revealed (Fig. 3d) the disappearance of the aldehyde proton signal at $\delta=9.9 \mathrm{ppm}$ and emergence of a new singlet at $\delta=8.4 \mathrm{ppm}$ corresponding to imine proton in 5 . This change was accompanied by the appearance of a new pair of aromatic doublets at $\delta=$ 6.8-7.2 ppm corresponding to the aromatic protons of imine 5 and the disappearance of the aromatic protons associated with aldehyde 3 . These observations suggest near-quantitative formation of imine 5 from aldehyde 3 and amine 2 at $\mathrm{pH} 12.0$ without the formation of any unwanted side-products. In particular, there is no evidence for the formation of unwanted hemithioacetal as observed by the absence of a singlet at $\sim \delta=$ 6.1-6.2 ppm. Because the increase in $\mathrm{pH}$ causes deprotonation of the aromatic thiol 4 resulting in thiolate $\mathbf{4}^{\prime}$, its aromatic signals appear as a pair of doublets at $\delta=6.7-7.2 \mathrm{ppm}$. It is also 
worthwhile to note that at $\mathrm{pH} 12$ aldehyde 3 is not susceptible to hydrate formation, as evidenced by the lack of a signal at $\sim \delta=6.0-6.1 \mathrm{ppm}$.<smiles>CCN(CC)CC(=O)Nc1ccc(SSc2ccc(NC(=O)CN(CC)CC)cc2)cc1</smiles><smiles>CC[NH2+]CNC(=O)c1ccc(S)cc1</smiles><smiles>CN(C)CCNC(=O)c1ccc(C=O)cc1</smiles><smiles>CCNCNC(=O)c1ccc([S-])cc1</smiles><smiles>CNCCNC(=O)c1ccc(/C=N/CCCOC)cc1</smiles>

The transition from node $\mathrm{D}$ to node $\mathrm{C}$ was completed by slow addition of $0.25 \mathrm{M} \mathrm{H}_{2} \mathrm{O}_{2}$ to drive the oxidation of the thiolate $4^{\prime}$ whilst maintaining $\mathrm{pH}$ at 11.8 . The resulting ${ }^{1} \mathrm{H}$ NMR spectrum (Fig. 3c) displays a pair of aromatic doublets at $\delta=7.3 \mathrm{ppm}$ corresponding to aromatic protons in $\mathbf{1}$ and the disappearance of the pair of doublets corresponding to the thiolate $4^{\prime}$. A singlet corresponding to the imine proton at $\delta=$ 8.4 ppm of 5 accompanied by the characteristic pair of aromatic doublets at $\delta=7.6 \mathrm{ppm}$ suggests the imine bond has been successfully retained. The presence of low-intensity doublets at $\sim \delta=8.0 \mathrm{ppm}$ suggest the presence of a very small fraction of unreacted aldehyde 3 . These observations indicate that successful oxidation of thiol 4 to form stable disulfide 1 at $\mathrm{pH} 11.8$ in the presence of imine 5 .

To ensure the complete reversibility of every step within the network, an anti-clockwise cycle was performed. ${ }^{1} \mathrm{H}$ NMR spectroscopic analysis of the system at each node displayed the expected resonances, suggesting that the system is reversible at each transition (see ESI $\dagger$ Fig. S4).

Taken together, these experiments demonstrate the high degree of orthogonality which can be displayed in the bond breaking and forming processes of disulfide and imine DCBs.

To further explore the limitations of orthogonality, additional experiments were performed suggesting that the disulfide and imine bonds are orthogonal only below $\mathrm{pH}$ 12.0. At $\mathrm{pH}$ values higher than 12.0 unwanted decomposition of disulfide $\mathbf{1}$ to yield the thiolate $\mathbf{4}^{\prime}$ occurs i.e. the stimuli which modulates the formation and hydrolysis of the imine bond is actually interfering with the disulfide bond. Direct attack by hydroxide resulting in cleavage of aromatic disulfide bonds has been reported by Danehy, ${ }^{12}$ and this undesired process sets an upper operational limit regarding $\mathrm{pH}$ upon the system.

Alternative reaction partners investigated by us failed to deliver orthogonality. When electron-rich aldehydes such as 4-hydroxybenzaldehyde were used, it was found that there is
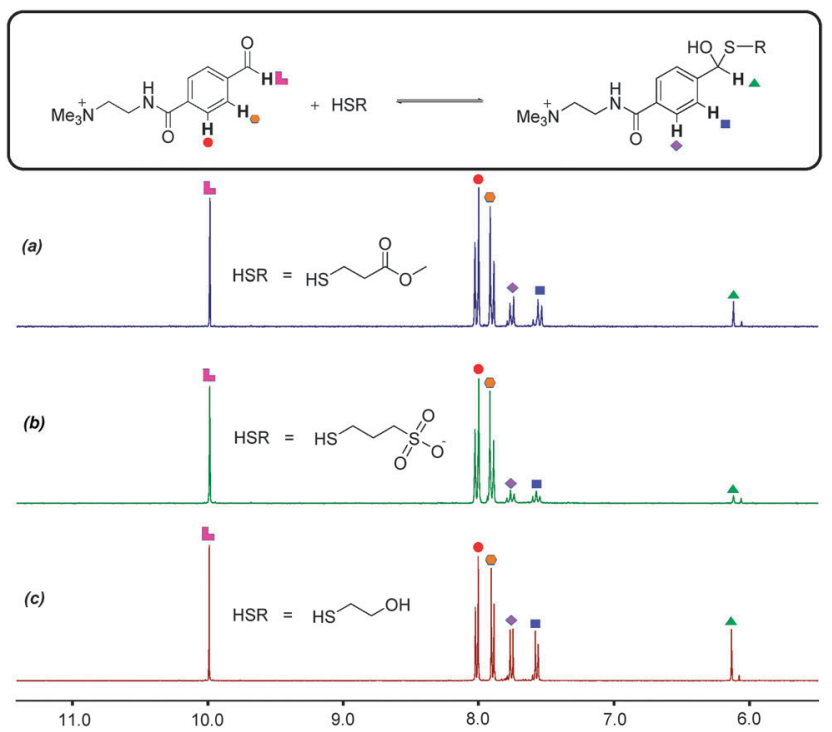

Fig. $4{ }^{1} \mathrm{H}$ NMR spectra $\left(300 \mathrm{MHz}, \mathrm{D}_{2} \mathrm{O}\right.$ ) demonstrating formation of hemithioacetal at $\mathrm{pH}$ 6.5. Aldehyde 3 and RSH present in $15 \mathrm{mM}$ concentration (30 mM total). RSH = (a) 2-mercaptoethyl acetate, (b) sodium 3-mercapto-1-propanesulfonate, (c) 2-mercaptoethanol. The low intensity singlet observed just upfield of the diagnostic hemithioacetal signal at $\delta=6.2 \mathrm{ppm}$ indicates the presence of expected hydrate formed by reaction the aldehyde with water.

very little reaction with amine 2 to form imine at $\mathrm{pH}$ 12.0. The chosen electron-deficient aromatic aldehyde 3 appears to be both relatively resistant to hydrate formation and is capable of forming imines at $\mathrm{pH}$ 12.0. A series of alkyl thiols were also investigated as substitutes for thiol 4. At $\mathrm{pH} 6.5$ in $\mathrm{D}_{2} \mathrm{O}$ these thiols engaged in nucleophilic attack at aldehyde 3 , with further ${ }^{1} \mathrm{H}$ NMR spectroscopic studies providing evidence for the formation of unwanted hemithioacetals (Fig. 4). The propensity to form hemithioacetals with electron-deficient aldehydes rules alkyl thiols out as potential thiol-disulfide system components. The chosen aromatic thiol $\mathbf{4}$ does not form hemithioacetal with aldehyde 3 , probably on account of aromatic thiols being poorer nucleophiles than alkyl thiols and thus less likely to form hemithioacetals.

In summary, our model investigation has shown the orthogonal nature of the bond-forming and bond-breaking processes of imine and disulfide DCBs. This study highlights the importance of carefully testing orthogonal systems through the application of wellconsidered models, and that careful choice of reaction partners is important to ensure the absence of any unwanted competing processes. We are now applying this knowledge to develop multistimuli responsive polymer materials.

We thank Dr Corinne Wills and Prof. William McFarlane for invaluable help and guidance with NMR spectroscopy.

\section{Notes and references}

$\$$ We follow the definition of orthogonality as proposed ${ }^{2}$ by Schmittel et al. as "two (or more) dynamic interactions without crosstalk", where without cross-talk denotes that "two or more interactions are mutually compatible and that alternative combinations are not detectable by means of the applied spectroscopic techniques". 
1 R. F. Ludlow and S. Otto, Chem. Soc. Rev., 2008, 37, 101-108.

2 M. L. Saha, S. De, S. Pramanik and M. Schmittel, Chem. Soc. Rev., 2013, 42, 6860-6909.

3 A. Wilson, G. Gasparini and S. Matile, Chem. Soc. Rev., 2014, 43, 1948-1962.

4 M. von Delius, E. M. Geertsema and D. A. Leigh, Nat. Chem., 2010, 2, 96-101.

5 M. A. A. Garcia and N. Bampos, Org. Biomol. Chem., 2013, 11, 27-30.

6 A. W. Jackson and D. A. Fulton, Macromolecules, 2012, 45, 2699-2708.

7 (a) W. Zhang, R. J. Denman, C. Yu and Y. Jin, Chem. Soc. Rev., 2013, 42, 6634-6654; (b) S. J. Rowan, S. J. Cantrill, G. R. L. Cousins, J. K. M. Sanders and J. F. Stoddart, Angew. Chem., Int. Ed., 2002, 41, 898-952.

8 J. M. Spruell and C. J. Hawker, Chem. Sci., 2011, 2, 18-26.

9 C. Godoy-Alcántar, A. K. Yatsimirsky and J. M. Lehn, J. Phys. Org. Chem., 2005, 18, 979-985.

10 B. T. Tuten, D. Chao, C. K. Lyon and E. B. Berda, Polym. Chem., 2012, 3, 3068-3071.
11 Although this work has a focus upon orthogonality in bond forming and bond breaking processes, it is important highlight that orthogonality of component exchange processes, which have been utilized within dynamic combinatorial libraries or other supramolecular systems, has previously been reported. For further information, see: (a) A. M. Escalante, A. G. Orrillo, I. Cabezudo and R. L. E. Furlan, Org. Lett., 2012, 14, 5816-5819; (b) A. M. Escalante, A. G. Orrillo and R. L. E. Furlan, J. Comb. Chem., 2010, 12, 410-413; (c) Z. RodriguezDocampo and S. Otto, Chem. Commun., 2008, 5301-5303; (d) S. P. Black, J. K. M. Sanders and A. R. Stefankiewicz, Chem. Soc. Rev., 2014, 43, 1861-1872; (e) M. Malakoutikhah, J. J.-P. Peyralans, M. ColombDelsuc, H. Fanlo-Virgós, M. C. A. Stuart and S. Otto, J. Am. Chem. Soc., 2013, 135, 18406-18417; $(f)$ S. Kulchat, K. Meguellati and J.-M. Lehn, Helv. Chim. Acta, 2014, 97, 1219-1236; $(g)$ M. Ciaccia, I. Tosi, L. Baldini, R. Cacciapaglia, L. Mandolini, S. Di Stefano and C. A. Hunter, Chem. Sci., 2015, 6, 144-151.

12 J. P. Danehy and K. N. Parameswaran, J. Org. Chem., 1968, 33, 568-572. 